\title{
DISSECTING ANEURYSM OF THE AORTA WITH RUPTURE INTO THE RIGHT ATRIUM
}

\author{
BY \\ MICHAEL DULAKE* AND RICHARD ASHFIELD $\dagger$ \\ From the Cardiac Department, Brompton Hospital, London
}

Rupture of the false sac in dissecting aortic aneurysm commonly occurs into the pericardial sac with an immediately fatal outcome (Hirst, Johns, and Kime, 1958). It is well known that should re-entry be established the immediate prognosis is not so grave as used to be thought, though death occurs later from heart failure due to aortic incompetence or hypertensive heart disease (Shennan, 1934). Dissection of the false sac back to the aortic root itself is common and rupture most frequently occurs into the pericardium (Gore and Seiwert, 1952). More rarely rupture into a cardiac chamber occurs. The right ventricle may be entered, a left-to-right shunt being established and, theoretically at least, this is amenable to surgical repair. This is more common with rupture of a sinus of Valsalva rather than a true dissection (Oram and East, 1955).

An aortic diastolic murmur is commonly found in true dissection. Various mechanisms for the murmur are described, such as aortic incompetence due to distortion of the valve ring, eddy currents in the distorted aorta, or ebb and flow into the false sac (Hirst et al., 1958).

In our case the false sac entered the right atrium. The signs of apparently florid aortic incompetence, however, obscured the signs of the left-to-right shunt though there was clinical evidence of this at least seven months before death. We can find no previously reported case where the sac has ruptured into the right atrium.

\section{Case Report}

The managing director of a light engineering firm, aged 49, was previously perfectly well until September 1 , 1960. Having gone to bed he developed sudden severe back pain between the scapulæ and also in the right leg which was also numb. The pain lasted half an hour, but for the three days following he was con. fined to bed at home with malaise and fever which progressively improved.

On convalescing he had considerable effort dyspnœa. On return home a loud systolic murmur was heard, which had not been previously noted, but his electrocardiogram was said to be normal. However, his erythrocyte sedimentation rate was raised. Blood urea and white count were reported as normal and two blood cultures were sterile. After these investigations in hospital he again went for convalescence.

Effort dyspnœa was worse than ever, being provoked by the slightest exertion which was accompanied by an ill-defined retrosternal discomfort radiating to the back and promptly relieved by rest. A persistent cough productive of frothy mucoid sputum occasioned his consulting a chest clinic physician who in turn referred him to Dr. C. G. Barnes at Hillingdon Hospital, where he was admitted on October 5, 1960.

The findings were now those of frank congestive cardiac failure. The cardiac impulse, of left ventricular type, was palpable in the fifth intercostal space, five inches from the mid-line. A slight lift over the right ventricular outflow was also noted. Carotid arterial pulsation was pronounced. An ejection murmur was audible over the whole præcordium, maximal at the lower end of the sternum. The femoral pulses were weak, the right being less than the left.

Dilatation of the ascending aorta was seen on fluoroscopy and the diagnosis of dissecting aneurysm was made. On salt restriction, digitalis, and diuretics he improved and was discharged home on November 5, 1960.

W.12.

* Present address: Department of Medicine (Cardiology), Hammersmith Hospital, Ducane Road, London

$\dagger$ Present address: Westminster Hospital, London S.W.1. 


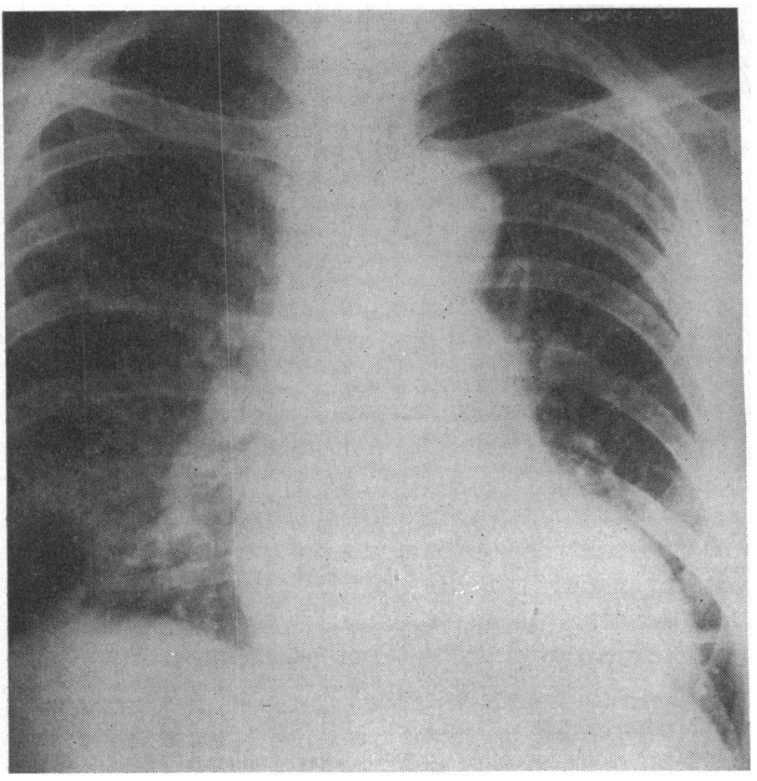

FIG. 1.-Chest radiograph showing enlargement of the right atrium, the main pulmonary artery and its right branch, in addition to the aorta and left ventricle.

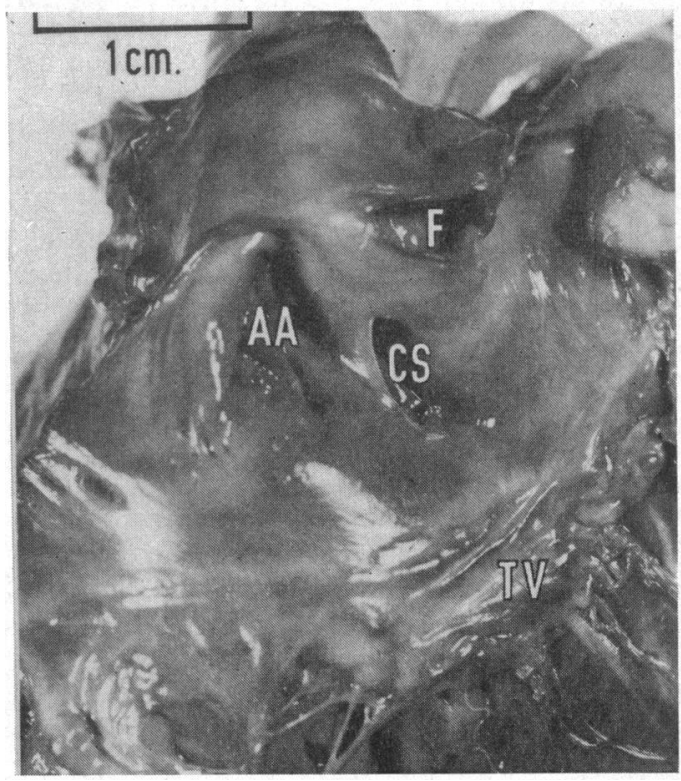

Fig. 2.-Close-up of the right atrium. The fistula (F) is seen above the opening into the coronary sinus (CS) and lies close to the atrial appendage (AA). The tricuspid valve (TV) is also shown.

He was reviewed by Dr. Barnes as an out-patient two weeks later, and a loud continuous murmur at the lower end of the sternum was elicited and a tentative diagnosis of an arteriovenous fistula was made. On this account he was referred to the late Dr. Paul Wood and admitted to Brompton Hospital on December 18, 1960.

He was ill, sallow, sweating, lethargic, and depressed. The findings were essentially as above except that the continuous murmur was not heard again. The venous pulse showed a conspicuous $6 \mathrm{~cm}$. "v" wave. The peripheral pulses, all present, were grossly collapsing, with atrial fibrillation

Chest radiograph (Fig. 1) showed widening of the aortic shadow, particularly of the knuckle where there was a double contour. There was considerable pulmonary venous engorgement which subsequently cleared. The electrocardiogram unexpectedly showed right ventricular hypertrophy, a vertical axis, and a dominant $\mathrm{S}$ in V5 with $\mathrm{T}$ wave inversion in leads V1 to V3, and confirmed atrial fibrillation.

Hæmoglobin and white blood count were normal and erythrocyte sedimentation rate was $7 \mathrm{~mm}$. in 1 hour (Westergren). Blood urea was $75 \mathrm{mg} . / 100 \mathrm{ml}$., falling to $13 \mathrm{mg}$. Wasserman and Kahn reactions were negative.

On vigorous treatment for heart failure, he slowly improved. He had intramuscular penicillin for three weeks ( $\frac{1}{2}$ mega unit crystalline every six hours) because one of two blood cultures yielded Streptococcus viridans. He had an occasional spike of fever up to $99 \cdot 8^{\circ} \mathrm{F} .\left(37 \cdot 7^{\circ} \mathrm{C}\right.$.) and an initial urine specimen showed moderate numbers of red cells.

The diagnosis was now aortic incompetence due to distortion of the valve sing by the aortic

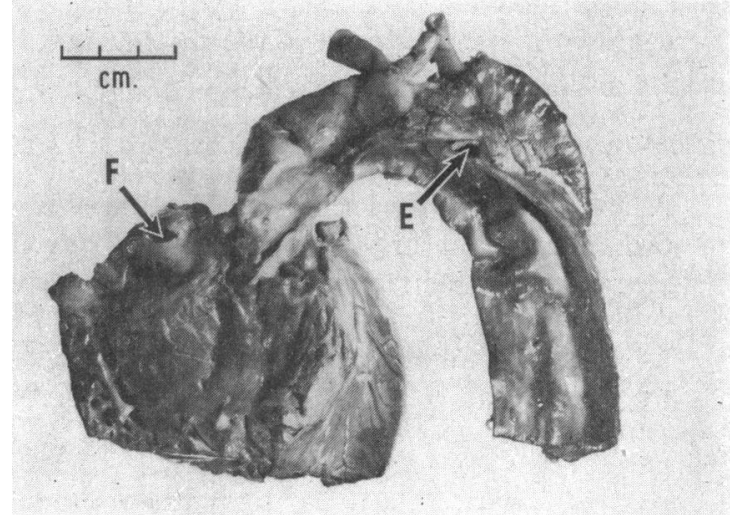

Fig. 3.-Part of the heart and aorta. The tear allowing entry into the false sac is shown (E) and the fistula into the right atrium (F). 
dissection. He was seen by Mr. M. Paneth with the possibility of surgical correction in mind, but it was felt that the risks were too great, and, as he was clinically improving on salt restriction, digoxin, and diuretics, operation was deferred.

He spent an uneventful convalescence and was reviewed in Dr. Wood's out-patient clinic on March 23. There was a gratifying improvement in the radiological appearances: the heart was considerably smaller and the pulmonary venous congestion had cleared. His effort tolerance, though not normal was much better.

However, in spite of continuing his treatment, there were some signs of deterioration at his visit on May 18, 1961. The possibility of surgical intervention was again discussed, but was again deferred. By July 13, 1961 , he had been back to work for six weeks without subjective ill effects, but the signs of left ventricular failure were again slightly increased. In addition to oral diuretics, digoxin, and salt restriction he was given mersalyl once weekly.

He was readmitted as an emergency on August 14, 1961, having deteriorated rapidly in the previous week, and died on the following day. The signs were, as before, those of florid aortic incompetence with the addition of cardiac and renal failure.

Post-mortem examination of the heart and great vessels (dissected by Mr. M. Paneth) revealed considerable dilatation of both ventricles. There was a $1 \mathrm{~cm}$. transverse rent in the aortic intima just distal to the origin of the left subclavian artery with dissection extending distally beyond the bifurcation of the common iliac vessels on both sides. Proximally the false sac had dissected back to the aortic valve ring though the internal diameter of the ring appeared normal and the valve cusps appeared normal and competent. However, a $5 \mathrm{~mm}$. button-hole communication with the right atrium was demonstrated entering the chamber just above the ostium of the coronary sinus. The margins of the fistula were clean and smooth and consistent with the communication having occurred some months previously (Fig. 2 and 3). There was moderate atheroma of the aorta, but the tear was not at the site of a plaque. Other findings were those of longstanding congestive cardiac failure.

\section{Discussion}

It is clear that we should have taken more note of the continuous murmur recorded by Dr. Barnes. In retrospect too, the electrocardiogram was out of keeping with aortic incompetence, for it had not the expected pattern of abnormal left ventricular dominance. Further, the peripheral lung fields were not merely congested, but had the appearance of plethora which, though lessening on treatment, never resolved. Cardiac catheterization could have demonstrated the left-to-right shunt at atrial level.

It was difficult enough to establish the true state of affairs at necropsy; to have elucidated this situation at thoracotomy would have been impossible. If a shunt had been found in life it might just have been possible to close it surgically, though the hazards of cardiopulmonary bypass requiring arterial cannulation would have been formidable. For this reason retrograde aortography would also have been contraindicated. A selective right ventricular or pulmonary artery angiogram would have shown re-opacification of the right atrium, but it is doubtful whether the exact site of the communication would have been visible.

\section{Summary}

A case of aortic dissection with rupture of the false sac into the right atrium is described. Complete diagnosis was not established in life as the signs closely resembled those of aortic incompetence due to the dissection. The possibility of surgical correction is discussed.

Thanks are due to Mr. M. Paneth for performing the cardiac dissection, to Dr. R. V. Gibson for permission to publish the case, and to Professor J. F. Goodwin for his helpful advice.

\section{References}

Gore, I., and Seiwert, V. J. (1952). Dissecting aneurysm of the aorta. Arch. Path., 53, 121.

Hirst, A. E., Johns, V. J., and Kime, S. W. (1958). Dissecting aneurysm of the aorta. A review of 505 cases. Medicine (Baltimore), 37, 217.

Oram, S., and East, T. (1955). Rupture of aneurysm of aortic sinus (of Valsalva) into the right side of the heart. Brit. Heart J., 17, 541.

Shennan, T. (1934). Dissecting Aneurysms. Spec. Rep. Ser. med. Res. Coun. (Lond.), No. 193. 